TRADUÇÃO

Heloísa Augusta Brito de Mello*

Dilys Karen ReEs*

A ETNOGRAFIA NA SALA DE AULA DE SEGUNDA LíNGUA: DEFININDO

O QUE É ESSENCIAL

Karen AnN Watson-Gegeo**

PRIMEIRAS PALAVRAS $* * *$

O artigo que aqui apresentamos é uma tradução do texto original de Karen Ann Watson-Gegeo, Ethnography in ESL: defining the Essentials, que tem por objetivo delinear os princípios essenciais da pesquisa etnográfica. Publicado em 1988 no TESOL Quarterly e posteriormente em 1995 na coletânea Readings on Second Language Acquisition, organizada por H. Douglas Brown e Susan T. Gonzo, o artigo destaca-se por apontar, de maneira sucinta e objetiva, uma opção metodológica bastante adequada para as pesquisas em sala de aula de segunda língua/língua estrangeira, uma linha de pesquisa em expansão no nosso Programa de Pós-Graduação. Esperamos que a tradução desse texto possa não só iluminar os caminhos dos nossos alunos pesquisadores iniciantes, mas também dar visibilidade a uma abordagem de pesquisa que tem sido muitas vezes negligenciada entre os nossos pares.

Sobre a autora

Karen Watson-Gegeo é professora titular da Faculdade de Educação da Universidade da Califórnia, Davis, onde ministra as disciplinas

* Professoras da Universidade Federal de Goiás.

** Watson-Gegeo, Karen Ann. Ethnography in ESL: defining the Essentials. In: Brown, H. Douglas e Gonzo, Susan T. Readings on second language acquisition. Englewood Cliffs, NJ: Prentice Hall, 1995. p. 36-54.

*** Agradecemos a autora, que gentilmente autorizou a tradução e a publicação deste artigo. 
Estudos Socioculturais e Língua, Letramento e Cultura. É também membro de Programas de Mestrado e Doutorado em Antropologia, Linguística, Desenvolvimento Humano e Desenvolvimento Comunitário e Regional. Anteriormente, lecionou por nove anos na Faculdade de Educação de Hayward, Harvard, e na Universidade do Havaí em Manoa por cinco anos. Foi pesquisadora do East-West Center por quatro anos, tendo sido responsável por projetos no sudeste da Ásia e nas Ilhas do Pacífico Sul. Desde 1978, Karen tem trabalhado em conjunto com David Welchman Gegeo em Kwara'ae on Mala'ita, nas Ilhas Salomão, uma parceria que tem resultado em inúmeras publicações sobre ensino-aprendizagem de línguas e culturas para crianças e sobre questões sociais tais como disputas por assentamentos rurais, aconselhamento familiar e matrimonial, desenvolvimento rural, e epistemologia, ontologia e filosofia indígena. Suas pesquisas estão ancoradas na teoria crítica (pós-estruturalista, pós-modernista e póscolonial) feminista e nas teorias desenvolvidas pelas minorias étnicas e por pesquisadores do terceiro mundo. Seu trabalho é de natureza multidisciplinar - antropologia, linguística, estudos culturais, ciência cognitiva, psicologia intercultural, desenvolvimento comunitário etc. e integra a etnografia crítica com análise do discurso e com socialização da linguagem.

\section{A tRADUÇão}

A tradução que aqui apresentamos tomou como referência o texto publicado na coletânea Readings on Second Language Acquisition em que os organizadores fazem uma pequena introdução ao texto de Karen denominada Antecipando o artigo. Nossa tradução procurou reproduzir o conteúdo, o estilo, a naturalidade e a fluência do texto original. Contudo, como acreditamos que toda tradução é fruto daquilo que consideramos ser o texto original, ou seja, da nossa interpretação do texto de partida, conforme argumenta Arrojo (1986, p. 44), assumimos as imprecisões que possam existir no texto traduzido.

Passamos, a seguir, à tradução propriamente dita. 
Antecipando o artigo

Nem toda pesquisa, quaisquer que sejam os meios, faz uso de estatística e de princípios da pesquisa experimental para obter conclusões significativas. Na verdade, numa área de estudo como a de aquisição de segunda língua em que (a) os fatores comportamentais humanos são difíceis de serem quantificados, e (b) as variáveis igualmente difíceis de serem controladas empiricamente é que vemos a necessidade de recorrer a outros paradigmas de pesquisa mais qualitativos.

Neste artigo, Karen Watson-Gegeo apresenta a etnografia, uma modalidade da pesquisa qualitativa. Os estudos etnográficos há anos têm sido usados nas áreas de antropologia e de educação, mas só recentemente se tornaram populares no campo da aquisição de segunda língua. Sugerimos que antes de iniciar a leitura do artigo, você procure encontrar respostas para as seguintes questões:

1. O que significa etnografia?

2. Qual é o propósito da autora? (Cf. o final da primeira seção.)

3. Faça suposições sobre como a pesquisa etnográfica se diferencia de outros métodos.

A etnografia como método de pesquisa para a investigação na sala de aula de segunda língua ou em outros contextos educacionais tornou-se popular recentemente. Contudo, muitos estudos denominados 'etnográficos' são impressionistas e superficiais ao invés de cuidadosos e detalhados. Este artigo focaliza duas questões: O que é etnografia? E o que a etnografia pode fazer em prol do ensino de segunda língua? Inicialmente, definimos etnografia e discutimos alguns princípios característicos de um trabalho etnográfico de qualidade, focalizando, sobretudo, o comportamento em grupos, a natureza holística, as perspectivas êmica-ética, a comparação, a teoria fundamentada em dados e as técnicas de coleta e tratamento dos dados. Em seguida, apresentamos a etnografia como uma opção para a pesquisa e a melhoria da qualidade do ensino e do treinamento de professores.

As pesquisas sobre a sala de aula de segunda língua, aquisição de segunda língua e educação bilíngue têm se apoiado em uma variedade de metodologias de pesquisa na última década ${ }^{1}$ (Cf. Allwright, 1983; 
Chaudron, 1986, 1987; Gaies, 1983; Long, 1980; Mitchell, 1985). No entanto, só recentemente, as metodologias de orientação etnográfica tornaram-se populares tanto na pesquisa educacional quanto na pesquisa em ensino de segunda língua. A etnografia foi acolhida com entusiasmo porque vislumbrava a possibilidade de se trazer alternativas de investigação para questões difíceis de serem tratadas por meio da pesquisa experimental como, por exemplo, os processos socioculturais na aprendizagem de línguas, as pressões institucionais e sociais que perpassam a interação na sala de aula a todo instante e como obter uma perspectiva mais holística das interações professor-aluno que auxilie na formação de professores e aperfeiçoe a prática de sala de aula.

Contudo, uma compreensão do que constitui um trabalho etnográfico científico de qualidade não acompanhou a crescente popularidade da etnografia no ensino de segunda língua. Para alguns, a etnografia tornouse sinônimo de pesquisa qualitativa de tal forma que qualquer abordagem qualitativa poderia ser chamada de 'etnográfica', parcialmente ou em seu todo, desde que envolvesse observação em ambientes não-laboratoriais. Alguns estudos qualitativos ou "naturalistas" são estruturados segundo esquemas codificados com base em categorias predeterminadas. Outros envolvem relatos impressionistas e períodos de observação reduzidos (ex. LightFoot, 1983). A natureza superficial de muitos estudos caricaturiza o ambiente de ensino-aprendizagem ao invés de caracterizá-lo, o que levou Rist (1980) a chamá-los de "etnografia relâmpago (blitzkrieg ethnography): ${ }^{2}$ o pesquisador "mergulha" no ambiente de pesquisa como num vôo rasante e faz observações a partir de categorias pré-estabelecidas ou observações totalmente impressionistas, e em seguida abandona o local para relatar os resultados apressadamente.

Se os relatos impressionistas não são etnográficos - e de fato não são-, o que é então etnografia? O que constitui um modelo metodológico para o estudo etnográfico? Por que deveríamos investigar o ensino e a aprendizagem de uma segunda língua etnograficamente?

O objetivo deste artigo é proporcionar uma visão panorâmica acerca das características essenciais à etnografia. Não é minha intenção criticar estudos já existentes ou fazer uma revisão exaustiva da literatura sobre etnografia. Ao invés disso, uma perspectiva metodológica etnográfica é esboçada, sugerindo formas de como a etnografia pode contribuir com o ensino e a aprendizagem de uma segunda língua. 
Originalmente desenvolvida na antropologia para descrever os "modos de vida" de um grupo social (HEATH, 1982), a etnografia é o estudo do comportamento das pessoas em contextos naturais e dinâmicos, com foco especial na interpretação cultural do comportamento (ver também FirTh, 1961; Hymes, 1982). O objetivo do etnógrafo é fornecer uma descrição e um relato interpretativo-explanatório do que as pessoas fazem em um determinado ambiente (por exemplo, uma sala de aula, a vizinhança, ou uma comunidade), do efeito de suas interações e da maneira que eles entendem o que estão fazendo (o significado que as interações têm para eles). Esta caracterização de etnografia, apesar de ser geral o bastante para incluir a maioria das formas de trabalho etnográfico, também é fiel a uma perspectiva antropológica. (Para discussões sobre diferentes tipos de trabalho etnográfico e sugestões de esquemas de classificação, confira Hymes, 1982; Werner \& SCHOEPFle, 1987.)

Os termos etnográfico, qualitativo e naturalista são frequentemente usados intercambiavelmente na literatura educacional, mas eles se diferem significativamente. Em seu sentido original, a pesquisa qualitativa se preocupa em identificar a presença ou ausência de algo e em determinar sua natureza ou aspectos peculiares (em contraste com a pesquisa quantitativa, que se preocupa com a quantificação dos dados). Pesquisa qualitativa é, pois, um termo guarda-chuva que abrange tipos diversos de abordagens e técnicas de pesquisa, incluindo a etnografia, os estudos de caso, a indução analítica, a análise de conteúdo, a semiótica, a hermenêutica, as histórias de vida e certos tipos de abordagens computacionais e estatísticas (KIRK \& MiLLER, 1986). Pesquisa naturalista é, por sua vez, um termo descritivo que implica a condução de observações pelo pesquisador em um "ambiente dinâmico e natural onde [as pessoas] vivem e trabalham" (Schatzman \& STRAuss, 1973, p. 5). A partir dessas definições, pode-se dizer que a etnografia é qualitativa e naturalística assim como muitos outros tipos de pesquisa qualitativa. A etnografia se diferencia das outras formas de pesquisa qualitativa no seu aspecto holístico e na maneira como trata a cultura como parte integral da análise (e não apenas como um dos vários fatores a serem levados em consideração). 
Para alcançar o objetivo de fornecer um relato descritivo, interpretativo e explanatório do comportamento das pessoas em um dado contexto, o etnógrafo faz observações sistemáticas, intensivas e detalhadas daquele comportamento, examinando como tal comportamento e interações são socialmente organizadas bem como as regras sociais, as expectativas interacionais e os valores culturais que subjazem ao comportamento.

Alguns princípios da pesquisa etnográfica

Aseguir, discutimos alguns dos princípios da pesquisa etnográficos já mencionados. Primeiro, a etnografia focaliza o comportamento de um grupo de pessoas e os padrões culturais subjacentes a esse comportamento. Por certo, os etnógrafos também se interessam pelos indivíduos, pois são os indivíduos que são observados e entrevistados e é entre eles que se desenvolvem os relacionamentos pessoais. Da mesma forma, as diferenças individuais são também importantes para o estabelecimento de variações no comportamento. Contudo, a maioria dos estudos etnográficos está preocupada com grupos ao invés de características individuais porque o comportamento cultural é por definição um comportamento compartilhado. Por exemplo, em um contexto de ensino de inglês como segunda língua um etnógrafo está mais focado no modo como a organização da sala de aula influencia o acesso do aluno aos diferentes tipos de insumo ou práticas que circulam na sala de aula do que nos problemas individuais de aprendizagem de língua. Quando os relatos etnográficos focalizam comportamentos individuais, o indivíduo é geralmente tratado como representativo do grupo. Um exemplo é o artigo de Carrasco (1981) que focaliza a organização social de uma sala de aula bilíngue, no qual ele ilustra como a colaboração entre professor-etnógrafo pode aguçar a percepção de algumas habilidades não-reconhecidas de determinadas crianças cujo professor provavelmente as "rotula" como "incapaz".

Segundo, a etnografia é holística, o que significa que qualquer aspecto da cultura ou do comportamento deve ser descrito e explicado em relação à totalidade do sistema do qual faz parte (DIESING, 1971; FIRTH, 1961). Por exemplo, um recorte da interação professor-aluno 
de uma aula de conversação em inglês pode ser visto como integrante de uma série de círculos concêntricos de contextos cada vez maiores ("macros"). Assim, se nos movemos de um micro-contexto de interação em direção a um macro, esses círculos podem incluir outras interações que ocorrem durante a aula, a sala de aula com suas características e restrições, a escola, a Secretaria de Educação (ou outros níveis da administração) e a sociedade.

Para descrever completamente um contexto de sala de aula seria necessário rastrear todos os sentidos e implicações que perpassam todos os contextos micro e macro teóricos que sejam descritivamente relevantes. Por exemplo, em um estudo etnográfico do processo de escrita dos alunos de uma sala multiétnica e urbana do sexto ano (CAzden, Michaels e Watson-Gegeo, Karen Ann, 1987), as atividades de escrita foram examinadas no contexto das demais atividades de sala, da formação dos professores, das culturas da família e da vizinhança, da organização social das escolas e lideranças, das implementações da Secretaria quanto ao processo de escrita, e do exame oficial de avaliação da escrita. Ulichny e Watson-Gegeo (no prelo) ${ }^{3}$ desenvolveram o estudo com o intuito de demonstrar a importância dos níveis institucionais e sociais de análise para explicar o discurso entre professor-aluno no que diz respeito à escrita.

Terceiro, a coleta de dados etnográficos tem início a partir de um referencial teórico que direciona a atenção do pesquisador para certos aspectos situacionais e para certos tipos de perguntas de pesquisa. $\mathrm{O}$ papel da teoria na condução da observação e interpretação da etnografia é frequentemente entendido de forma equivocada quando fora da antropologia. Os etnógrafos não afirmam que entram em um contexto de pesquisa como uma tabula rasa, isto é, sem conceitos prévios ou diretrizes para a observação. A teoria é importante para ajudar os etnógrafos a decidir quais evidências são plausíveis de se tornarem significativas para responder às questões de pesquisa postas no início do estudo e desenvolvidas no campo (NARRol e CoHEN, 1970, 1973; Pelto e Pelto, 1970). Se a observação não é guiada por um referencial teórico explícito, ela será guiada somente pela ontologia implícita do observador, isto é, por seus valores, atitudes e suposições acerca de "que tipo de coisas constitui o mundo [ou o universo do estudo], sobre como elas se relacionam e agem" (DIESING, 1971, p. 124). 
Em uma etnografia da sala de aula, a literatura de pesquisa inclui, em geral, vários estudos detalhando as características da organização e da interação em sala de aula; identificando e analisando padrões, tipologias ou modelos de interação (por exemplo, a sequência instrucional tripartite de Mehan [1979]; ver também Mehan, 1982) e/ ou relacionando-os a fatores institucionais, sociais e culturais (por exemplo, Boggs, 1985; MCDERMotT \& Hood, 1982; Ulichny \& WATSONGegeo, impresso). Embora cada sala de aula seja um contexto e uma situação única, esses estudos se destacam porque desenvolveram um vocabulário conceitual para examinar padrões de organização social e de interação (ex. atividades de fala [LEvinson, 1979], estruturas de participação [PHILIPS, 1972], rotinas [WATSON, 1975] que direcionam a atenção do etnógrafo para maneiras nas quais o comportamento é tipicamente organizado ou estruturado em sala de aula e por que. Deste modo, esses estudos proporcionam embasamento teórico para comparação entre contextos distintos e para as decisões iniciais do etnógrafo sobre o quê observar.

Embora guiada pela teoria existente (especialmente a de base etnográfica), a observação e a interpretação etnográfica não são por ela determinadas. Por um lado, cada situação investigada por um etnógrafo deve ser entendida em si mesma. No que diz respeito ao processo de pesquisa, isso significa que o etnógrafo muda o foco de observação para incluir fenômenos e interações fora do escopo sugerido pela teoria consultada, tanto para corrigir os equívocos da teoria quanto para investigar interações, padrões de comportamentos e outros fenômenos significativos ou talvez únicos à situação estudada. Compreender uma situação em seus próprios termos está intimamente relacionado com a geração de teoria fundamentada (discutida abaixo).

Além disso, cada situação investigada por um etnógrafo deve ser entendida a partir da perspectiva dos participantes naquela situação específica. Essa última característica da pesquisa etnográfica é denominada princípio êmico-ético de análise, para o qual nos voltamos agora.

ANÁliSe E COMPARAÇão DOS CONCEITOS ÊMICO-ÉTICO

A partir dos significados dos termos fonêmico/fonético oriundos da linguística, Pike (1964) criou a distinção êmico-ético para se referir 
aos significados culturais. Pike enfatiza que o termo êmico ou o enquadre culturalmente específico usado pelos membros de uma sociedade/ cultura para interpretar e dar significados às experiências vividas diferem em várias maneiras do enquadre ontológico ou interpretativo do pesquisador (enquadre ético) (cf. HyMEs, 1982).

As análises e interpretações éticas se baseiam no uso de enquadres, conceitos e categorias da linguagem analítica das ciências sociais e são potencialmente úteis para a pesquisa comparativa entre línguas, contextos e culturas. Entretanto, para serem adequados de tal forma, devem ser cuidadosamente definidos e operacionalizados. Deste modo, as categorias fonéticas de "diferenças distintivas" são relativamente estáveis e, portanto, úteis para análises interlinguísticas. Talvez o caso clássico da terminologia ética seja as denominações antropológicas de parentesco que se referem aos relacionamentos de natureza biológica (ex. irmão da mãe) ao invés daqueles que se referem aos relacionamentos sociais (ex. tio). $\mathrm{O}$ termo em inglês tio pressupõe obrigações e comportamentos específicos à cultura americana ou britânica que não são compartilhados com outras culturas.

Entretanto, a terminologia ética, em raras situações é culturalmente neutra, uma vez que a sua origem é geralmente a cultura do pesquisador ou aquilo que se pode chamar de "cultura da pesquisa" propriamente dita (neste caso, referindo-se às tradições e às formas discursivas que evoluíram em certas disciplinas de pesquisa). Por exemplo, categorias usadas em esquemas codificadores não-etnográficos acerca da interação em sala de aula são, nesse sentido, frequentemente problemáticos (ex. o sistema Flanders e seus descendentes). Atitude, correção, elogio, pergunta complexa, iniciação, sentimentos de aceitação assim como outros termos ou frases similares têm sido usados com frequência nas pesquisas de sala de aula de segunda língua em geral e de inglês como segunda língua. Além do problema da inconsistência em definir e operacionalizar tais categorias (CHAUDRON, 1986) e do problema da verificação dessas categorias que em geral mascaram a natureza contingente da interação (MeHAN, 1981), tais termos juntamente com suas definições operacionais, podem ou não ter validade para os professores e alunos cujos comportamentos são classificados ou avaliados. 
A preocupação quanto ao entendimento que os participantes têm das situações nas quais eles são observados tem levado os etnógrafos a enfatizar análises êmicas. Como indicado acima, o termo êmico referese às perspectivas, interpretações e categorias culturalmente baseadas e que são usadas pelos membros do grupo estudado para conceitualizar e codificar conhecimento, além de guiar o próprio comportamento. Termos, conceitos e categorias êmicos são, portanto, funcionalmente relevantes à investigação dos comportamentos das pessoas estudadas pelo etnógrafo. Uma análise construída em conceitos êmicos incorpora as perspectivas do participante e as interpretações do comportamento, dos eventos e das situações por meio de uma linguagem descritiva verbatim (cf. SPRADLEY, 1979).

É importante reconhecer que uma análise êmica não faz uma mera substituição dos termos usados na linguagem e no contexto do participante por aqueles usados pelo pesquisador. Por exemplo, uma análise não é êmica se simplesmente substituir professora por teacher ao se referir à pessoa cuja profissão é instruir outras pessoas porque o contexto estudado é uma escola em Porto Rico. Ao contrário, o que é importante sobre a distinção teacher-profesora é que os dois termos são parte de enquadres distintos, envolvendo quais papéis a instrutora tem em relação aos seus alunos, o que ela espera dos alunos e o que eles esperam dela em termos de obrigações e comportamentos mútuos, expectativas da sociedade em relação às responsabilidades da instrutora e assim por diante. Essas diferenças devem fazer parte da análise.

A análise etnográfica não é exclusivamente êmica. Ao contrário, uma análise êmica cuidadosa deve preceder e constituir a base para uma análise ética que permita comparações interculturais ou intercontextuais. O quarto princípio ou característica da pesquisa etnográfica é a sua natureza comparativa (FIRTH, 1961). O etnógrafo procura inicialmente construir uma teoria específica ao contexto estudado para só então fazer comparações ou generalizações com outros contextos ou situações investigados de modo semelhante. As comparações devem ser construídas a partir de uma perspectiva êmica cuidadosa, levando-se em conta que uma comparação equilibrada entre aspectos de dois ou mais contextos é praticamente impossível. Contudo, é possível fazer comparações num nível mais abstrato. 
Por exemplo, nosso interesse particular está no desenvolvimento de estratégias culturalmente apropriadas para a sala de aula como uma "ponte" de mediação para os alunos bilíngues, de minorias e de Terceiro Mundo cujo conhecimento linguístico e/ou cultural prévio difere daquele da escola. Um exemplo bem sucedido é o Programa de Educação Infantil Kamehameha (KEEP), em que os resultados dos exames nacionais de leitura de crianças da primeira série de uma sala de aula experimental foram significativamente melhores após a introdução de atividades de leitura baseadas em eventos de fala da comunidade havaiana denominados "contar-estórias". Uma característica chave de contar-estórias é a co-narração, a apresentação conjunta de experiências pessoais, informação e interpretação de eventos por dois ou mais contadores. Os pesquisadores do programa KEEP estruturaram as lições de leitura a partir do formato contar-estórias para criar o que Boggs (1985) chama de "contar estórias com um livro" (p. 139) e fizeram mudanças culturalmente baseadas na organização da sala de aula (Au e Jordan, 1891; BogGS, 1985; veja também WATsOn, 1975; Watson-Gegeo e BogGs, 1977).

A experiência KEEP inspirou nosso trabalho atual com comunidades rurais nas Ilhas Salomão onde as crianças iniciam o jardim de infância em classes de imersão e apresentam altos índices de reprovação (WATSON-GEgeo e Gegeo, 1988).Como etnógrafo, nossa expectativa era não encontrar um formato equivalente para o evento contar-estórias (parte de um enquadre êmico havaiano) nas Ilhas Salomão, mas que descobríssemos um evento semelhante que, como contar-estórias, poderia ser adaptado para uso em sala de aula.

Contudo, parece-nos agora que os eventos de fala denominados nas Ilhas Salomão "moldando-a-mente" pode ser a opção acertada. Como evento de fala, moldando-a-mente envolve o ensino intensivo da língua, comportamento adequado, formas de raciocínio e conhecimento cultural ministrados em sessões especiais caracterizadas por um ambiente sério, um registro formal e discussões com argumentação. Tendo em vista que um importante propósito das atividades moldandoa-mente é o ensino com foco nas habilidades linguísticas, (especialmente a conscientização de vocabulário e habilidades metalinguísticas [WATSON-GEGEO, 1987]), pode ser bastante útil se adaptado para uso nas salas de aula de imersão de língua inglesa. Moldando-a-mente se baseia 
em uma abordagem êmica de ensino diferente tanto dos modelos de ensino havaianos de contar-estórias quanto dos americanos/ocidentais (Watson-Gegeo, 1986; Watson-Gegeo e Gegeo, 1988).

Como eventos de fala contrastantes e que se baseiam em assunções culturalmente diferentes, os eventos contar-estórias e moldando-a-mente do Havaí e das Ilhas Salomão, respectivamente, são conceitos êmicos, e não éticos ou diretamente comparáveis. Entretanto, em um nível mais abstrato, pode-se falar em estratégias de ensino culturalmente apropriadas e fazer a comparação entre estudos etnográficos acerca de tais estratégias, como elas se relacionam com outros aspectos das culturas nas quais são encontradas (isto é, valores, teorias de aprendizagem locais, estruturas sociais, instituições), como elas podem ser adaptadas à pedagogia da sala de aula e até que ponto elas são eficazes em termos de melhores resultados no desempenho.

Todos os exemplos usados para ilustrar os princípios da pesquisa etnográfica assumem um modelo de aprendizagem de língua interativo. A perspectiva etnográfica acerca do ensino de línguas é a da socialização da linguagem ao invés da aquisição da linguagem (COOK-GuMPERz, CORSARO e Streeck, 1986; Schieffelin e Ochs, 1986; Watson-Gegeo, no prelo). A substituição da socialização por aquisição coloca a aprendizagem de línguas em um domínio de socialização mais compreensivo, a experiência de vida em que os indivíduos aprendem desde cedo os significados culturais e como desempenhar habilidades, tarefas, papeis e identidades esperadas em quaisquer sociedades em que eles venham viver (Wuthnow, Hunter, Bergesen e Kurzweil, 1984, p. 40).

A perspectiva da socialização da linguagem implica que a língua é aprendida por meio da interação social. Isso significa que a língua é o veículo primário da socialização: quando aprendemos uma segunda língua, aprendemos mais do que uma estrutura para comunicação; também aprendemos (por exemplo) normas sociais e culturais, procedimentos de interpretação e formas de raciocínio. $\mathrm{O}$ estudo etnográfico da socialização da linguagem direciona, portanto, a atenção do pesquisador não apenas para o ensino e a aprendizagem ou a aquisição de habilidades linguísticas, mas também para o contexto de aprendizagem ou para qualquer outro aspecto (valores, atitudes, enquadres de interpretação) que é aprendido e ensinado ao mesmo tempo com a estrutura linguística. 
Como produto, a etnografia é uma descrição detalhada e uma análise de um contexto social e da interação que nele ocorre. Um contexto social pode ser uma sala de aula, uma instituição, um bairro ou uma comunidade, sendo que esses contextos podem ser definidos em níveis diversos de inclusão social. Uma etnografia completa oferece uma teoria fundamentada em dados (Glaser \& Strauss, 1967) do contexto e da sua respectiva cultura. Por fundamentada entende-se uma teoria baseada em e derivada de dados e obtida por meio de um processo sistemático de indução. A teoria fundamentada em dados pode ser tanto substancial (isto é, focada em um tópico empírico como a interação professor-aluno que ocorre na sala de aula de segunda língua) ou formal (com foco em um tópico conceitual como, por exemplo, um modelo para a aquisição de segunda língua) (DiesInG, 1971, P. ; Glaser \& STRAUSS, 1967, p. 32).

Como método, a etnografia inclui as técnicas de observação, a observação-participante (observação e interação simultânea com os participantes do estudo), as entrevistas formais e informais com os participantes do estudo em situações reais, os registros em áudio ou vídeo para análises verticalizadas das interações, coleta de documentos e materiais relevantes disponíveis no contexto de pesquisa, bem como outras técnicas necessárias para responder às perguntas de pesquisa de um determinado estudo. Historicamente, os etnógrafos têm adotado metodologias ecléticas, recorrendo a métodos de pesquisas tanto quantitativos quanto qualitativos quando for o caso (Pelto e Pelto, 1970). Nos últimos 15 anos, a análise do discurso (de vários tipos) tornou-se uma abordagem central para a análise dos dados nos trabalhos etnográficos (Boggs, 1985; Cazden, John e Hymes, 1972; Heath, 1983; Mehan, 1979; Trueba, Guthrie e Au, 1981).

Uma das características do método etnográfico é a observação detalhada e intensiva de um contexto durante um longo período de tempo. Idealmente, um etnógrafo que observa uma sala de aula de inglês como segunda língua de nível universitário deveria observar todas as aulas que ocorrem durante todo o semestre, realizar entrevistas com uma amostra dos alunos e com o professor e, se possível, observar os alunos em outros contextos. Se o etnógrafo está estudando a aquisição 
de uma segunda língua em uma dada comunidade, ele ou ela deveria fazer uma amostragem sistemática dos locais, dos participantes, dos eventos, dos horários e dos tipos de interação que ocorrem no contexto por meio de observações e entrevistas ao longo de vários meses ou até mesmo anos. As escolhas dos contextos, das situações e do tamanho das amostras para observação e entrevistas dependem das perguntas de pesquisa e dos objetivos do estudo.

Os etnógrafos não fazem uso listas de categorias previamente fixas e quantificáveis em seus formatos de observação porque tais instrumentos não podem capturar a complexidade da interação em sala de aula e nem lidar com a relação entre o comportamento verbal e o não verbal ou entre o comportamento e o contexto (MeHAN, 1981, p. 39). Além disso, a maioria dos formatos de codificação já existentes envolve pequenos períodos de observação recorrente, por exemplo, quando uma folha de codificação é marcada a cada 3 segundos por 2 minutos em intervalos de 10 minutos ao longo de uma hora de observação na sala de aula. Tais unidades arbitrárias de tempo de observação não conseguem captar todas as interações que ocorrem durante os vários minutos ou períodos mais longos e que, portanto, mascaram o uso funcional da língua e a complexidade da interação (GLEASON, no prelo).

Um estudo etnográfico de longa duração projetado para um ano ou mais envolve normalmente três estágios de trabalho, a saber: estágio de abrangência, estágio orientado pelo tópico e estágio orientado pelas hipóteses (tomando aqui emprestado os termos de HyMEs [1982]). No estágio de abrangência, o etnógrafo estuda todos os aspectos teóricos importantes de um contexto, conduzindo uma ampla gama de observações, com mapeamento do local, levantamento censitário e entrevistas. Por exemplo, em uma sala de aula bilíngue, o etnógrafo observa as atividades da sala de aula que ocorrem durante todo o dia e conduz observações em diversos micro-contextos da escola, tais como na sala dos professores, no pátio e no refeitório durante o almoço (ver GuTHRIE, 1985). Todos esses locais podem fornecer informações acerca das habilidades bilíngues das crianças, suas atitudes em relação ao uso e repertório linguístico e outras informações acerca de alunos, professores e o envolvimento dos pais na escola. A triangulação - procedimento de juntar as informações oriundas de diferentes fontes de dados e/ou de 
coletar dados por meio de diferentes metodologias de pesquisa tais como observação participante, entrevistas e mapeamento de rede de pesquisas e survey (FIELding \& Fielding, 1986) - é uma estratégia importante na validação dos resultados de um trabalho etnográfico (Diesing, 1981).

Durante o estágio orientado pelo tópico, o etnógrafo se concentra no esclarecimento e afunilamento do tópico central do estudo por meio de observações focalizadas, entrevistas e análise dos dados já coletados. O etnógrafo se concentra na descrição cuidadosa da interação e eventos tais como eles ocorrem no contexto, com o propósito de gerar questões de pesquisa focadas e/ou hipóteses. Os dados coletados até então na forma de notas de campo feitas com base nas observações in loco são codificados segundo categorias, a partir das interações recorrentes e relevantes para o surgimento das questões de pesquisa. Gravações em áudio e vídeo são transcritas e registradas com o auxílio das notas de campo, e quando a interação é o foco da pesquisa, uma análise preliminar do discurso deve ser feita.

O estágio orientado pelas hipóteses se baseia na verificação das hipóteses e tem o propósito de responder as perguntas de pesquisa por meio de observações focadas posteriores, de entrevistas verticalizadas (geralmente estruturadas), de análise contínuas do discurso e outras formas de análise sistemática (cf. Werner \& Schoepfle, 1987). Algumas das hipóteses a serem consideradas durante este estágio podem ser oriundas da literatura, outras das observações, entrevistas ou análises. Este estágio pode incluir quantificação na forma de contagem frequencial (frequency counts), testes de significância, ou análise multivariada de padrões e temas (tais como em dados discursivos; cf. JАCOB, 1982).

Entre o conjunto de orientações metodológicas proposto por Mehan (1979) para pesquisa etnográfica está o tratamento holístico dos dados (comprehensive data treatment), geralmente ausente dos estudos denominados etnográficos em ensino de segunda língua e educação. Desse modo, Mehan sugere que a análise deve ser conduzida de forma a contemplar todo o material ou dados coletados. Uma das falhas em muitos estudos publicados é o seu apoio em alguns poucos relatos usados para sustentar o ponto de vista teórico ou as conclusões do pesquisador, mas geralmente escolhidos com critérios não muito claros para os leitores (por exemplo, PRESTON, 1981, parece equiparar relatos esparsos 
com etnografia). Quando exemplos são usados como ilustrações em um relatório etnográfico, eles devem resultar de uma seleção sistemática de exemplos representativos, em que tanto as variações quanto a tendência principal ou a recorrência dos dados são refletidas. Qualquer outra coisa pode caricaturizar ao invés de caracterizar o que o etnógrafo observou e registrou. (cf. a discussão de WeRnER e SCHOEPFLe, 1987, acerca dos 28 padrões mínimos para o desenho e análise da pesquisa etnográfica).

\section{UsOS DA PESQUISA ETNOGRÁFICA NO ENSINO DE SEGUNDA LíNGUA}

Por que estudar o processo de ensino e aprendizagem de uma segunda língua etnograficamente? Porque a etnografia é uma alternativa bastante significativa dentre outras formas de se pesquisar a educação tendo em vista que permite lidar com questões essenciais relativas à teoria e prática.

Uma dessas questões essenciais diz respeito ao que ocorre cada momento em contextos de ensino e aprendizagem de segunda língua. $\mathrm{O}$ ensino de segunda língua acontece em um amplo espectro de contextos institucionais, incluindo salas de aula de inglês como língua estrangeira no Japão, salas bilíngues no Novo México, salas de aula específicas de inglês como segunda língua (pullout classes) para alunos refugiados do sudeste asiático nos estados do centro-oeste americano. A aprendizagem de uma segunda língua ocorre em um espectro ainda mais amplo, incluindo os contextos familiares e comunitários. Contudo, até agora poucos são os estudos que caracterizam esses contextos assim como a interação ensino-aprendizagem que neles ocorrem (BREEN, 1985).

Os métodos etnográficos nos oferecem uma abordagem para uma documentação sistemática da interação ensino-aprendizagem com detalhes ricos e contextualizados com o objetivo de desenvolver uma teoria melhor fundamentada (ou seja, uma teoria gerada a partir dos dados coletados). Essa é uma alternativa para as pesquisas do tipo "descendente" (top down) baseadas em modelos pré-existentes que possam mascarar características importantes acerca de contextos que não tenham sido estudos previamente (GLASER \& STRAuss, 1967). Por se caracterizar como uma pesquisa de longo prazo, a pesquisa etnográfica permite uma investigação de como o ensino e outros padrões de 
interação se desenvolvem e mudam ao longo do tempo em um dado contexto. Essa perspectiva dinâmica é uma alternativa que permite corrigir a natureza estática das pesquisas que se apóiam em observações ou testes únicos.

A pesquisa etnográfica faz-nos lembrar da importância da cultura no ensino e aprendizagem de uma segunda língua, além de nos oferecer uma opção para o tratamento dessa questão. Em relação à cultura e ao ensino, por exemplo, é possível recorrer à etnografia para investigar o papel do professor no que diz respeito a como esse papel é definido e aceito nas diversas sociedades. No Japão, o ensino é uma atividade de prestígio e respeito, o relacionamento professor-aluno é respeitoso e formal, e a responsabilidade da aprendizagem está centrada no aluno ao invés de estar centrada no professor (White, 1987). Nos Estados Unidos, os professores não gozam de tal prestígio ou respeito e cada vez mais se espera que eles atendam às necessidades dos alunos. Os americanos esperam que os professores assumam a função de pais substitutos e quase sempre são vistos como os culpados pelo baixo desempenho dos alunos.

Tais diferenças em relação às expectativas da sociedade mostram que o que é considerado um comportamento adequado entre professor e aluno varia substancialmente de um contexto cultural para outro. $\mathrm{O}$ conselho que oferecemos aos professores de língua inglesa em formação - tais como ser amigável, carinhoso e generoso com os alunos - pode ser inapropriado em alguns contextos culturais se tomarmos como referência um conceito americano (por exemplo, o professor que compartilha detalhes de sua vida pessoal com os alunos teria uma atitude inapropriada em muitas sociedades asiáticas). Uma pesquisa etnográfica pode documentar e analisar o que se estabeleceu como boas relações entre professores e alunos num dado contexto cultural ou escolar de modo que essa informação fique disponível para o treinamento de professores.

Segundo, com relação à cultura e aprendizagem, a pesquisa na área da psicolinguística enfatiza a importância da teoria de esquemas para a aprendizagem. A etnografia chama a atenção para o fato de que muitos dos esquemas são culturalmente baseados e possíveis de serem estudados por meios etnográficos (cf. QuinN \& Holland, 1986).

Outra contribuição importante da etnografia para o ensino de segunda língua e para a pesquisa educacional, mencionada anteriormente, é a análise do contexto escolar institucional juntamente com as 
pressões sociais que alunos e professores sofrem (OGBU, 1974, 1978). São poucos os estudos que focalizam em como as pressões sociais e institucionais afetam a sala de aula de segunda língua ou dialetal. $\mathrm{O}$ estudo realizado por Guthrie (1985) acerca da educação bilíngue em uma comunidade de americano-chineses ilustra o papel complexo que tem a escola do município, as políticas federais, o apoio aos diferentes tipos de programas bilíngues e as divisões na comunidade local quanto ao debate entre programas bilíngues transicionais ou de manutenção. Cleghorn \& Genesee (1984) demonstram claramente as ligações entre os fatores educacionais e sociais que afetam os objetivos do programa e os resultados de um estudo etnográfico de um programa de imersão francês em Montreal.

De modo semelhante, os métodos etnográficos podem ser usados para estudar as pressões sociais e institucionais que afetam as inovações educacionais de forma inesperada por aqueles que as desenvolveram. Dois exemplos são: o uso pedagógico de computadores na sala de aula e no processo de ensino da escrita. O estudo de Cazden et al (1987), mencionado anteriormente, mostra que em duas salas de aula de sexta série estudadas durante um período superior a dois anos, os professores acomodaram os microcomputadores na rotina da sala de aula existente, ao invés de usarem os computadores para mudar a maneira como a escrita era ensinada. O resultado foi que os microcomputadores passaram a ser usados como máquinas de escrever eletrônicas para digitar a versão final dos textos, ao invés de editar e revisar as primeiras versões.

As restrições institucionais e da sala de aula que criaram essa situação também afetaram a introdução de uma abordagem de escrita como processo nessas salas. Como estavam sendo institucionalmente pressionados para terem seus alunos aprovados nos testes de escrita de final de ano, os professores concentraram seus esforços nos erros mecânicos dos alunos que apareciam nas primeiras versões escritas. Conferências sobre escrita (com a intenção de auxiliar as crianças a focalizar as idéias e a desenvolver uma consciência acerca de bons padrões de escrita) passaram a ser eventos de correção orientada (Ulichny e Watson-GegeO, no prelo; cf. também ZAMEL, 1985).

A abordagem holística da pesquisa etnográfica nos permite integrar a pesquisa em leitura com a pesquisa em escrita para tratar tanto da aquisição quanto do letramento. O estudo etnográfico exaustivo 
de Heath (1983) acerca dos usos da leitura e da escrita nos lares das crianças negras e brancas da classe trabalhadora e em comunidades das Carolinas do Norte e do Sul mostrou a importância de se compreender as experiências prévias dos aprendizes com a língua e com as práticas de letramento. Tal entendimento é crítico para os padrões de um ensino eficaz de inglês padrão (cf. SPOLSKY, 1082). Seu estudo tem implicações importantes não somente para o ensino de falantes de variedade não-padrão, mas também para o ensino de inglês para imigrantes e refugiados. Estudos etnográficos em outros contextos podem auxiliar professores de inglês como segunda língua que trabalham em outros países a antecipar as necessidades de seus alunos em relação às suas experiências anteriores de letramento e às suas maneiras de pensar com o objetivo de desenvolver materiais de ensino-aprendizagem adequados. Os estudos etnográficos podem também ajudar os professores a compreenderem as expectativas que seus alunos têm acerca da sala de aula e de seus estilos de interação.

A etnografia permite ao professor se beneficiar tanto da própria prática etnográfica quanto da aplicação dos resultados de pesquisa. Primeiro, técnicas etnográficas de observação e de entrevistas podem ser usadas para supervisão e feedback tanto para o treinamento inicial de professores quanto para o desenvolvimento do corpo docente. Em razão de as técnicas de observação etnográficas adotarem uma perspectiva holística acerca do comportamento contextualizado e também em razão de o etnógrafo buscar alcançar uma compreensão êmica das interações, as técnicas etnográficas podem ser usadas para fornecer aos professores um feedback acerca do que ocorre na sala de aula, incluindo as interações que escapam ao controle do professor ou o comportamento do professor que foge ao seu próprio controle. Hymes (1981) refere-se a esse tipo de avaliação feita pelos professores como "monitoramento etnográfico" da sala de aula. Carrasco (1981), mencionado anteriormente, faz uso de seus dados dessa maneira com importantes resultados tanto para os alunos quanto para o professor. Outros etnógrafos-professores enfatizam o valor da relação colaborativa professores-pesquisadores na operacionalização direta de mudanças positivas para a qualidade do ensino e da aprendizagem na sala de aula (por exemplo, Florio e WALSh, 1981; GrimMETt e GRANGER, 1983). 
Segundo, a etnografia pode ajudar os professores a fazerem a diferença em suas salas de aula. Os professores podem aprender a metodologia de pesquisa etnográfica formalmente por meio de um curso sobre etnografia ou informalmente por meio de um estágio colaborativo com algum etnógrafo experiente. Ao aperfeiçoar a habilidade de observação, os professores podem visualizar novas percepções acerca da organização da sala de aula, das estratégias de ensino e aprendizagem e dos padrões interacionais de suas próprias salas de aula. Essas observações se tornam, então, a base para a reflexão dos professores sobre suas próprias práticas e para a experimentação de técnicas alternativas de ensino e de gerenciamento de sala de aula. A combinação de pesquisa etnográfica intensiva em sala de aula e de observações etnográficas de professores sobre sua própria prática pode produzir compreensões múltiplas do que Richards (1987) chama de ensino bom.

Além disso, os professores podem envolver seus alunos no fazer etnográfico nas suas próprias comunidades e posteriormente usar os materiais desenvolvidos pelos alunos por meio de observações e entrevistas como uma base para a aprendizagem de habilidades de escrita (HeAth, 1983).

\section{CONCLUSÃo}

Nosso objetivo neste artigo foi o de mostrar que o verdadeiro trabalho etnográfico é sistemático, detalhado e rigoroso ao invés de anedótico e impressionista. A vantagem da etnografia para a pesquisa em segunda língua, para o treinamento de professores e para as práticas de sala de aula está na ênfase holística, na descrição densa e nas análises das interações entre professores e aprendizes e nos vários níveis de contexto em que essas interações ocorrem. É importante que a pesquisa denominada etnografia seja realmente etnográfica, o que significa que ela deve ser conduzida segundo os mesmos padrões de sistematicidade e rigor esperados daquelas pesquisas de qualidade desenvolvidas na área das ciências sociais. 


\section{NOTAS}

1 N. do T.: A autora faz referência à década de 80 .

2 N. do T.: O Blitzkrieg (termo alemão para guerra-relâmpago) foi uma doutrina militar em nível operacional que consistia em utilizar forças móveis em ataques rápidos e de surpresa, com o intuito de evitar que as forças inimigas tivessem tempo de organizar a defesa. Seus três elementos essenciais eram o efeito surpresa, a rapidez da manobra e a brutalidade do ataque.

3 Hoje o texto está publicado conforme a referência: Polly Ulichny; Karen Ann Watson-Gegeo. Interactions and authority: The dominant interpretive framework in writing conferences. Discourse Processes, v. 12, Issue 3 July 1989, p. 309-328.

\section{REFERÊNCIAS}

Allwright, D. Classroom-oriented research on language teaching and learning: a brief historical overview. TESOL Quartely, 17, 1983. p. 191-204.

Arrojo, R. Oficina de tradução: a teoria na prática. São Paulo: Ática, 1986. p. 44.

Au, K. H. P. \& JoRdan, C. Teaching Reading to Hawaiian children: finding a culturally appropriate solution. In: Trueba, H. T.; Guthrie, G. P. \& K. H. P. A (Eds.). Culture and the bilingual classroom: studies in classroom ethnography. Rowley, MA: Newbury House, p. 139-152.

BogGs, S. T. (with the assistance of K. A. Watson-Gegeo \& G. MacMillan). Speaking, relating and learning: a study of Hawaiian children at home and at school. Norwood, NJ: Ablex, 1985.

BREEN, M. P. The social context for language learning - a neglected situation? Studies in second language acquisition, 7, 1985. p. 135-158.

Calkins, L. M. Andrew learns to making writing hard. Language Arts, 56, 1979. p. 569-576.

CARRASCO, R. L. Expanded awareness of student performance: a case in applied ethnographic monitoring in a bilingual classroom. In: TRUEBA, H. T.; GUTHRIE, G. P. \& K. H. P. A (Eds.). Culture and the bilingual classroom: studies in classroom ethnography. Rowley, MA: Newbury House, 1981. p. 153-177.

Cazden, C. B.; John, V. P. \& Hymes, D. (Eds.). Functions of language in the classroom. New York: Columbia University, Teachers College Press, 1972. 
Cazden, C. B.; Michaels, S. \& Watson-Gegeo, K. A. Final report: microcomputers and literacy project (Grant n. G-83-0051). Washington, DC: National Institute of Education, 1987.

Chaudron, C. The interaction of quantitative and qualitative approaches to research: a view of the second language classroom. TESOL Quarterly, 20, 1986. p. 709-717.

Chaudron, C. Second language classrooms: research on teaching and learning. Cambridge: Cambridge University Press, 1987.

Cleghorn, A. \& Genesee, F. Language in contact: an ethnographic study of interaction in an immersion school. TESOL Quarterly, 18, 1984. p. 595-625.

Cook-Gumperz, J.; Corsaro, W. \& Streeck, J. (Eds.). Children's worlds and children's language. The Hague: Mouton, 1986.

Diesing, P. Patterns of discovery in the social science. Chicago: Aldine, 1971.

Fielding, N. G. \& Fielding, J. L. Linking data. Beverly Hills, CA: Sage, 1986.

FIRTH, R. Elements of social organization. Boston: Beacon, 1961.

Florio, S. \& WAlSh, M. The teacher as colleague in classroom research. In: Trueba, H. T.; Guthrie, G. P. \& Au, K. H. P. (Eds.). Culture and the bilingual classroom: studies in classroom ethnography. Rowley, MA: Newbury House, 1981. p. 87-104.

GAIES, S. J. The investigation of language classroom processes. TESOL Quarterly, 17, 1983.205-217.

Glaser, B. \& Strauss, A. The discovery of grounded theory. Chicago: Aldine, 1967.

Gleason, J. J. Special education in context: an ethnographic study of persons with developmental disabilities. New York: Cambridge University Press. (in press).

Grimmett, S. A. \& GRANGeR, C. Resolution in context: a teacher-researcher partnership. Educational Horizons, 61, 1983. p. 94-97.

Guthrie, G. P. A school divided: an ethnography of bilingual education in a Chinese community. Hillsdale, NJ: Lawrence Erlbaum, 1985.

Heath, S. B. Ways and words: language, life, and work in communities and classrooms. Cambridge: Cambridge University Press, 1983.

Hymes, D. Ethnographic monitoring. In: Trueba, H. T.; Guthrie, G. O. \& $\mathrm{Au}, \mathrm{K}$. H. P. (Eds.). Culture and bilingual classroom: studies in classroom ethnography. Rowley, MA: Newbury House, p. 56-68. 
Hymes, D. What is ethnography? In: Gilmore, P. \& Glatthorn, A. A. (Eds.). Children in and out of school: Ethnography and education. Washington, DC: Center for Applied Linguistics, 1982. p. 21-32.

$\mathrm{J}_{\mathrm{ACOB}}$, E. Combining ethnographic and quantitative approaches: suggestions and examples from a study on Puerto Rico. In: Gilmore, P. \& Glatthorn, A. A. (Eds.). Children in and out of school: Ethnography and education. Washington, DC: Center for Applied Linguistics, 1982. p. 124-147.

KIRK, J. \& MILER, M. L. Reliability and validity in qualitative research. Beverly Hills, CA: Sage. 1986.

Levinson, S. Activity types and language. Linguistics, 17, 1979. p. 363-369.

LightFоoт, S. L. The good high school: portraits of character and culture. New York: Basic Books, 1983.

LoNG, M. Inside the 'black box': Methodological issues in classroom research on language learning. Language Learning, 30, 1980. p. 1-42.

MCDERMOTT, R. P. \& Hood, L. Institutionalized psychology and the ethnography of schooling. In: Gilmore, P. \& Glatthorn, A. A. (Eds.). Children in and out of school: Ethnography and education. Washington, DC: Center for Applied Linguistics, 1982. p. 232-249.

Mehan, H. Learning lessons: social organization in the classroom. Cambridge, MA: Havard University Press, 1979.

Menan, H. Ethnography of bilingual education. In: Trueba, H. T.; Guthrie, G. P. \& Au, K. H. P. (Eds.). Culture and the bilingual classroom: studies in classroom ethnography. Rowley, MA: Newbury House, 1981. p. 36-55.

Menan, H. The structure of classroom events and their consequences for student performance. In: Gilmore, P. \& GlatThorn, A. A. (Eds.). Children in and out of school: Ethnography and education. Washington, DC: Center for Applied Linguistics, 1982. p. 232-249.

Mitchell, R. Process research in second-language classrooms. Language Teaching, 18, 1985. p. 330-362.

Narroll, R., \& Cohen, R. A handbook of method in cultural anthropology. New York: Natural History Press, 1970.

NARroll, R., \& Cohen, R. Main currents in ethnological theory. New York: Apple-ton-Century-Crofts, 1973.

OGBu, J. U. The next organization: an ethnography of education in an urban neighborhood. New York: Academic Press, 1974. 
Ogbu, J. U. Minority education and caste: the American system in crosscultural perspective. New York: Academic Press, 1978.

Pelto, P. \& Pelto, G. Anthropological research: the structure of inquiry. New York: Harper \& Row, 1970.

PhILIPS, S. U. Participant structures and communicative competence: Warm Springs children in community and classroom. In: CAzDEN, C. B.; John, V. P. \& Hymes, D. (Eds.). Functions of language in the classroom. New York: Columbia University, Teachers College Press, 1972. p. 370-394.

PIKE, K. L. Language in relation to a unified theory of structures of human behavior. The Hague: Mouton, 1964.

Preston, D. R. The ethnography of TESOL. TESOL Quarterly, 15, 1981. p. 105-116.

Quin, N. \& Holland, D. (Eds.). Cultural models in language and thought. Cambridge: Cambridge University Press, 1986.

RichARDS, J. C. The dilemma of teacher education in TESOL. TESOL Quarterly, 21, 1987, p. 209-226.

Rist, R. Blizkrieg ethnography: on the transformation of a method into a movement. Educational researcher, v. 9, n. 2, p. 8-10, 1980.

Schatzman, L. \& Strauss, A. L. Field research: strategies for a natural sociology. Englewood Cliffs, NJ: Prentice-Hall, 1973.

Schieffelin, B. B. \& Ochs, E. Language socialization. Annual Review of Anthropology, v. 15, p. 161-191, 1986.

Spolsky, B. Sociolinguistics of literacy, bilingual education, and TESOL. TESOL Quarterly, 16, 1982. p. 141-151.

Spradley, J. P. The ethnographic interview. New York: John Wiley, 1979.

Trueba, H. T.; Guthrie, G. P. \& Au, K. H. P. (Eds.). Culture and the bilingual classroom: studies in classroom ethnography. Rowley, MA: Newbury House, 1981.

Ulichny, P. \& Watson-Gegeo, K. A. (in press). Interactions and authority: the dominant interpretive framework in writing conferences. Discourse Processes.

Watson, K. A. Transferable communicative routines: strategies and group identity in two speech events. Language in society, 4, p. 53-70, 1975.

Watson-Gegeo, K. A. Heavy words and important silences: the social transfer of cognitive skills in Kwara'ae. Paper presented at the third international conference on thinking, Honolulu, January, 1987. 
Watson-Gegeo, K. A. \& Boggs, S. T. From verbal play to talk story: the role of routines in speech events among Hawaiian children. In: ERVIN-TRIPP, S. \& Mitchell-Kernan, C. (Eds.). Child discourse. New York: Academic Press, 1977. p. 67-90.

Watson-Gegeo, K. A. \& Gegeo, D. W. Schooling, knowledge and power: social transformation in the Solomon Islands. University of Hawaii working papers in ESL, v. 7, n. 1, p. 119-140, 1988.

Werner, O. \& Schoepfle, G. M. Systematic fieldwork, v. 1. Foundations of ethnography and interviewing, v. 2. Ethnographic analysis and data management. Newbury Park, CA: Sage, 1987.

Whiтe, M. The Japanese educational challenge: a commitment to children. New York: Free Press, 1987.

Wuthnow, R.; Hunter, J. D.; Bergesen, A. \& Kurzweil, E. Cultural analysis. Boston: Routledge \& Kegan Paul, 1984.

ZAMEL, V. Responding to student writing. TESOL Quarterly, 19, 1985. p. 79101. 